\title{
On small scale magnetic structures in the solar photosphere
}

\author{
S. Stangl and J. Hirzberger
}

\author{
Institut für Geophysik, Astrophysik und Meteorologie, Universitätsplatz 5, 8010 Graz, Austria \\ e-mail: stefan.stangl@uni-graz.at
}

Received 9 July 2004 / Accepted 3 November 2004

\begin{abstract}
The distribution of the magnetic field on the solar surface is as yet unknown in detail, but of considerable importance for solar physics in general. We have observed two different solar regions, one containing a small pore, the other region comprising a network in the light of the Fe I $\lambda 6301.5 \AA$ and Fe I $\lambda 6302.5 \AA$ lines at the German Vacuum Tower Telescope of the Observatorio del Teide (Tenerife, Spain) with the "Göttingen" Fabry-Pérot Interferometer. By applying image reconstruction techniques to broad- and narrowband filtergrams we obtained continuum images, line core images, as well as line-of-sight velocity and magnetic field maps. We present scatter plots of the line core intensity vs. the Doppler velocity and the vertical component of the magnetic field, which reveal that the line core brightness is not a clear indicator for magnetic fields in the solar atmosphere. Furthermore, we estimate the swaying motion of flux tubes to be mostly smaller than 0.3 and thus in good agreement with the predictions of theoretical dynamic models. Finally, we show how the choice of the observed solar target influences the results and their interpretation. We claim that generalizations can mislead, and strongly depend on the presence or absence of solar (magnetic) features in the analyzed data.
\end{abstract}

Key words. Sun: photosphere - Sun: magnetic fields - techniques: spectroscopic - techniques: polarimetric

\section{Introduction}

Analysis of the magnetic field distribution in the solar atmosphere is a challenging task since the theoretically predicted properties of magnetic structures hypothesise a large variety of features which cannot be resolved by existing solar instruments. Thus, none of the present theoretical models, i.e. on the one hand the microstructuring hypothesis developed by Sánchez Almeida (1997) and on the other hand the magnetopause model by Steiner (2000), can be verified because of the lack of the required angular resolution. Therefore, we are coerced into observing the seeing-degenerated magnetic configurations on the Sun by means of present telescopes and techniques, with the improvements kept at the back of our minds.

The high field strength of magnetic elements at small scales was deduced by Stenflo (1973), while their size of smaller than $200 \mathrm{~km}$ was inferred by Dunn \& Zirker (1973) from $\mathrm{H} \alpha$ filtergrams. These results were confirmed by means of high resolution spectrograms and magnetograms in the visible range (e.g. Title et al. 1992; Stolpe \& Kneer 1997) and in the infrared where the spectral lines are completely split by the Zeeman effect (e.g. Zayer et al. 1989; Muglach \& Solanki 1992). Recently, Wiehr et al. (2004) measured the spatial sizes of bright points from $G$-band images obtained at the Swedish Solar Telescope, and found the most frequent value for the diameters to be $160 \mathrm{~km}$. The continuum brightness of magnetic flux concentrations was determined to be $20 \%$ to $40 \%$ above the continuum intensity by observations (Keller 1992) and by simulations (Steiner et al. 1998), however, a reasonable filling factor for the interpretation of observational data has to be assumed (Schüssler \& Solanki 1988).

The correlation of the magnetic field, continuum intensity, and velocity in the photosphere was previously studied by Montagne et al. (1996) (hereafter: MMV96) using photographic spectra of the Fe I $\lambda 6301.5 \AA$ and Fe I $\lambda 6302.5 \AA$ lines. They find small bright flux tubes in the intergranular lanes and larger magnetic structures darker than the mean photospheric intensity. For field strengths lower than $400 \mathrm{G}$ downflow velocities of at most $200 \mathrm{~m} \mathrm{~s}^{-1}$ were measured. These results are qualitatively in good agreement with those of Title et al. (1992) (hereafter: TTT92) and Topka et al. (1992). More recently, Leka \& Skumanich (1998) presented bivariate correlations of the normalized continuum intensity vs. the magnetic field magnitude and the Doppler velocity for evolving magnetic regions. They detect a mostly linear relation of the intensity to the magnetic field magnitude and a small velocity dispersion $\left(\lessgtr \pm 0.5 \mathrm{~km} \mathrm{~s}^{-1}\right)$ in the case of a region where a pore is formed in a previous quiet Sun field.

The present work aims at the correlation of physical quantities such as the line-of-sight magnetic field strength and the Doppler velocity at small spatial scales. Thus this study provides insight in the properties of magnetohydrodynamic fine structures, which is needed to understand the detailed magnetic field distribution of flux tubes at small scales of the photosphere.

In the second section of this paper we describe the observation as well as the optical setup of the campaign. The third 


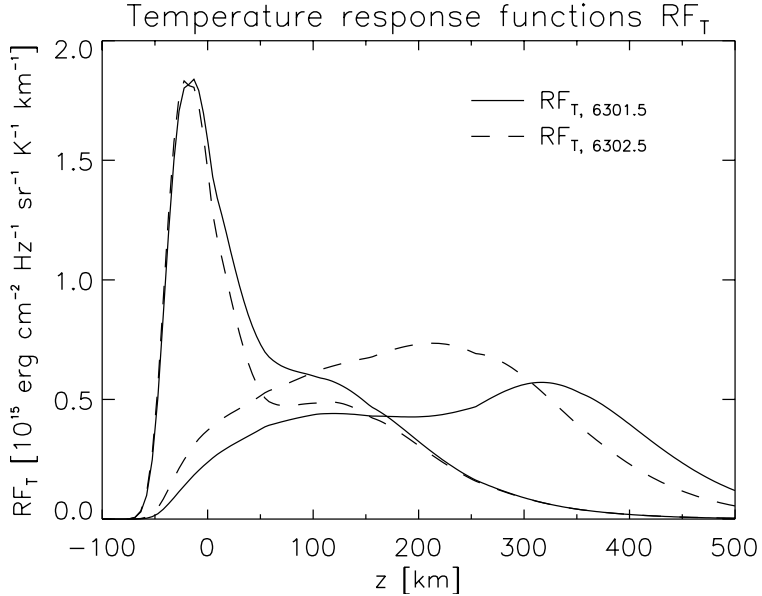

Fig. 1. Convolved temperature response functions of Fe I $\lambda 6301.5 \AA$ (solid lines) and Fe I $\lambda 6302.5 \AA$ (dashed lines) computed for a VAL$\mathrm{C}$ model atmosphere. The continuum response functions are rather narrow around $z=0 \mathrm{~km}$ and look quite similar, while the line core curves have lower maxima and are broader for both wavelengths.

section concentrates on the data processing and the determination of the physical quantities (line-of-sight (LOS) magnetic field strength, $B_{\mathrm{LOS}}$, Doppler velocity maps, $v_{\mathrm{LOS}}$, and line core (LC) images, $I_{\mathrm{LC}}$ ). The results are presented in the fourth section and conclusions are drawn in the last section.

\section{Observations}

The observations were carried out with the "Göttingen" FabryPérot Interferometer (FPI) of the German Vacuum Tower Telescope (VTT) of the Observatorio del Teide (Tenerife, Spain), with which we scanned the Fe I $\lambda 6301.5 \AA$ and the Fe I $\lambda 6302.5 \AA$ line. The FPI was adjusted to have a Full Width Half Maximum (FWHM) of $44 \mathrm{~m} \AA$ for the operating wavelength. The chosen fields of view (FOV) included a region with a small pore and a network region containing several bright points, which were selected by means of a video camera using a G-band filter and line core images of the Fe I $\lambda 6302.5 \AA$ line where some of these bright points are easy to identify.

The optical setup for the observations with the "Göttingen" FPI is extensively described in Bendlin et al. (1992), Bendlin \& Volkmer (1995), and Koschinsky et al. (2001). The light beam is divided by a beam splitter. One part is led through a broadband filter of $\sim 100 \mathrm{~m} \AA F W H M$, centered roughly at $6300 \AA$, to the broadband CCD camera, while the other beam passes the FPI and the Stokes $V$ polarizer and is then detected by the narrowband camera. Both CCD cameras have $384 \times 286$ pixels and the size of a pixel element is 0 ' $^{\prime} 1 \times 00^{\prime} 1$. They are operated in a strictly simultaneous mode since this is important for the reconstruction of the data. The broadband data provide the basis for the post-processing speckle reconstruction, while the narrowband data contain the scans through the spectral lines and thus yield the Stokes $I \pm V$ profiles.

In order to estimate the line formation heights of the two iron lines we computed temperature response functions (RFs) with a code described in Pérez Rodríguez \& Kneer (2002). The $R F \mathrm{~s}$ are shown in Fig. 1 for both continuum (peaks at
Table 1. List of observed spectral lines and corresponding formation heights.

\begin{tabular}{lcccccc}
\hline \hline Element & $\begin{array}{c}\lambda \\
{[\AA]}\end{array}$ & $g_{\text {eff }}$ & $z_{\text {cont }}$ & $z_{\text {LC }}$ & $\Delta z$ & $z_{\mathrm{LC}}^{*}$ \\
& {$[\mathrm{~km}]$} & {$[\mathrm{km}]$} & {$[\mathrm{km}]$} & {$[\mathrm{km}]$} \\
\hline $\mathrm{Fe} \mathrm{I}$ & 6301.508 & $5 / 3$ & 80 & 253 & 173 & 371 \\
$\mathrm{Fe} \mathrm{I}$ & 6302.499 & $5 / 2$ & 80 & 212 & 132 & 308 \\
\hline
\end{tabular}

approximately $-20 \mathrm{~km}$ ) and line cores (curves with lower maxima) as calculated for the VAL-C model atmosphere (Vernazza et al. 1981). The Fe I $\lambda 6301.5 \AA$ line response functions are displayed as solid lines, whereas the Fe I $16302.5 \AA R F \mathrm{~s}$ are printed as dashed lines. To obtain the corresponding formation heights we computed the center-of-gravity (COG) of each $R F$. All $R F$ s were convolved by an Airy function of $44 \mathrm{~m} \AA F W H M$. This convolution affects primarily the line center $R F$ s which are strongly broadened, whereas the continuum formation heights remain almost unaffected. Table 1 lists the laboratory wavelengths $\lambda$, the effective Landé factors $g_{\text {eff }}$, the continuum $\left(z_{\text {cont }}\right)$ and line core formation heights $\left(z_{\mathrm{LC}}\right)$, the height difference $(\Delta z)$, and the values for the non-broadened $R F \mathrm{~s}$ of the line cores $\left(z_{\mathrm{LC}}^{*}\right)$. From Table 1 one determines the two continuum formation heights as $80 \mathrm{~km}$, hence the continua form in the same photospheric layer. In contrast, the line core formation height values are $253 \mathrm{~km}$ ( $\mathrm{Fe}$ I $\lambda 6301.5 \AA$ ) and $212 \mathrm{~km}$ (Fe I $\lambda 6302.5 \AA$ ), respectively. Their COG heights vary by about $40 \mathrm{~km}$, so we have contributions from slightly different layers in our line core data. However, note that the line core $R F \mathrm{~s}$ are very broad and span a height range of approximately $600 \mathrm{~km}$, thus there are considerable contributions from lower layers of the photosphere.

The Fe I $\lambda 6301.5 \AA$ line was sampled at 14 , the Fe I 16302.5 $\AA$ line at 11 and an adjacent telluric oxygen line (6302.764 $\AA$ ) at 5 spectral positions with 5 images at each position, therefore we obtained 150 images at a total of 30 wavelength positions. The distance between two wavelength steps was set to $31.74 \mathrm{~m} \AA$ and the time needed for one scan was $51 \mathrm{~s}$ : $35 \mathrm{~s}$ for the sampling of the lines and an additional $16 \mathrm{~s}$ for the storage on hard disks. On May 11, 2002 we recorded two time series. The first consists of 32 scans, resulting in some $26 \mathrm{~min}$, and hereafter its FOV is referred to as Region 1 (cf. Fig. 2). The seeing was good during the first 24 scans (Fried parameter $r_{0} \geq 9.8 \mathrm{~cm}$ ), however, it dropped below this value for the rest of the series. The second time series contains 40 scans, thus it covers 34 min. This FOV is defined as Region 2 (see Fig. 3). Unfortunately, the resolution of the images did not improve until the second half of the observation run, i.e. $r_{0}$ was larger than $9.8 \mathrm{~cm}$ for the last 20 scans (corresponding to $17 \mathrm{~min}$ ) and thus limiting the length of the time series.

Both observed regions were located near the disk center: Region 1 at a heliocentric angle $\mu=0.98$ and Region 2 at $\mu \approx$ 1.00 . 


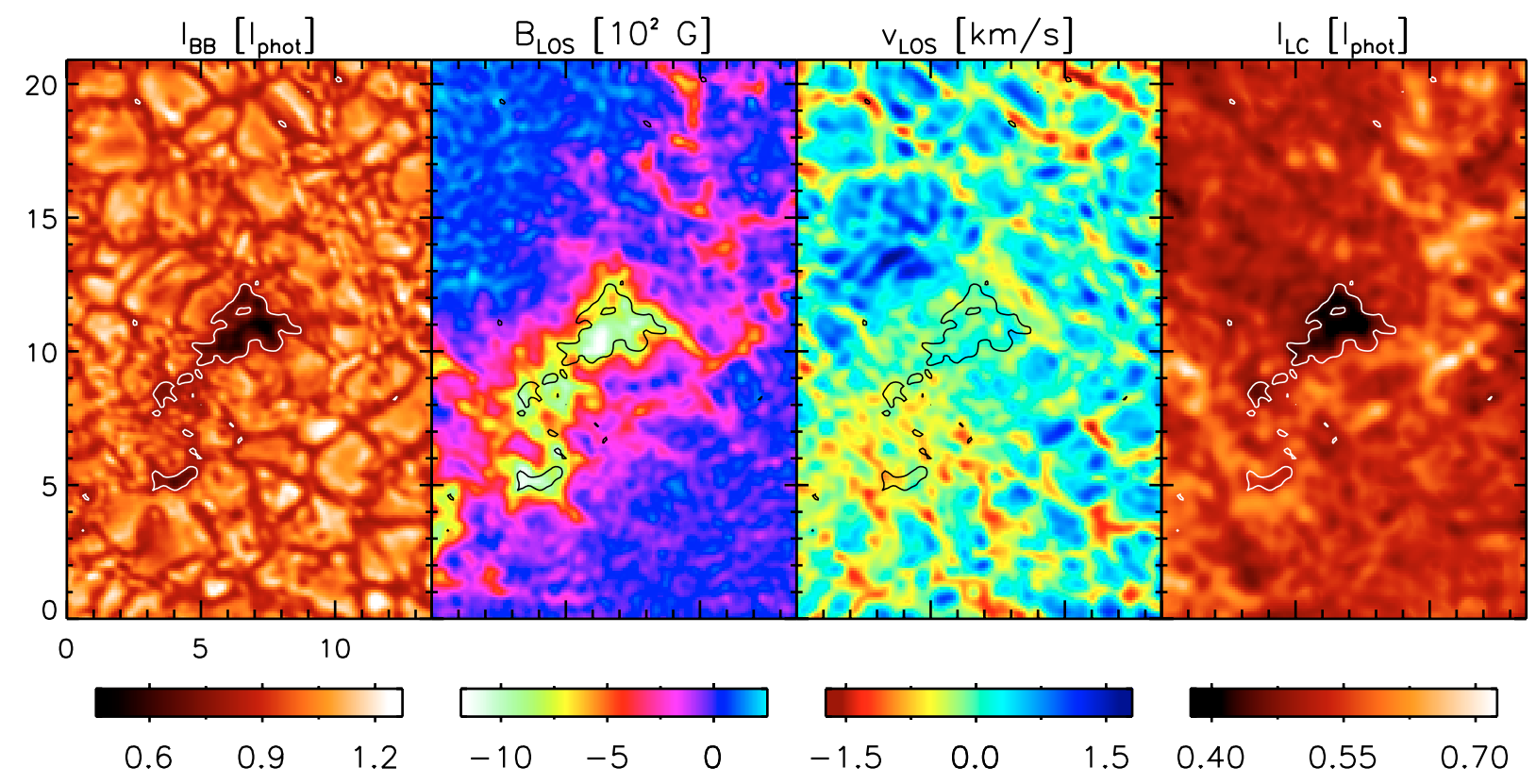

Fig. 2. Region 1: from left to right: speckle-reconstructed broadband image, $I_{\mathrm{BB}}$, line-of-sight magnetic field map, $B_{\mathrm{LOS}}$, line-of-sight velocity image, $v_{\mathrm{LOS}}$, and line core map, $I_{\mathrm{LC}}$. The field of view is approximately $14^{\prime \prime}$ by $21^{\prime \prime}$ and the tick marks are at distances of $1^{\prime \prime}$. The displayed maps are computed from the results of the Fe I $\lambda 6301.5 \AA$ A measurements. The overplotted contours outline a broadband intensity of $0.77 I_{\text {phot }}$.

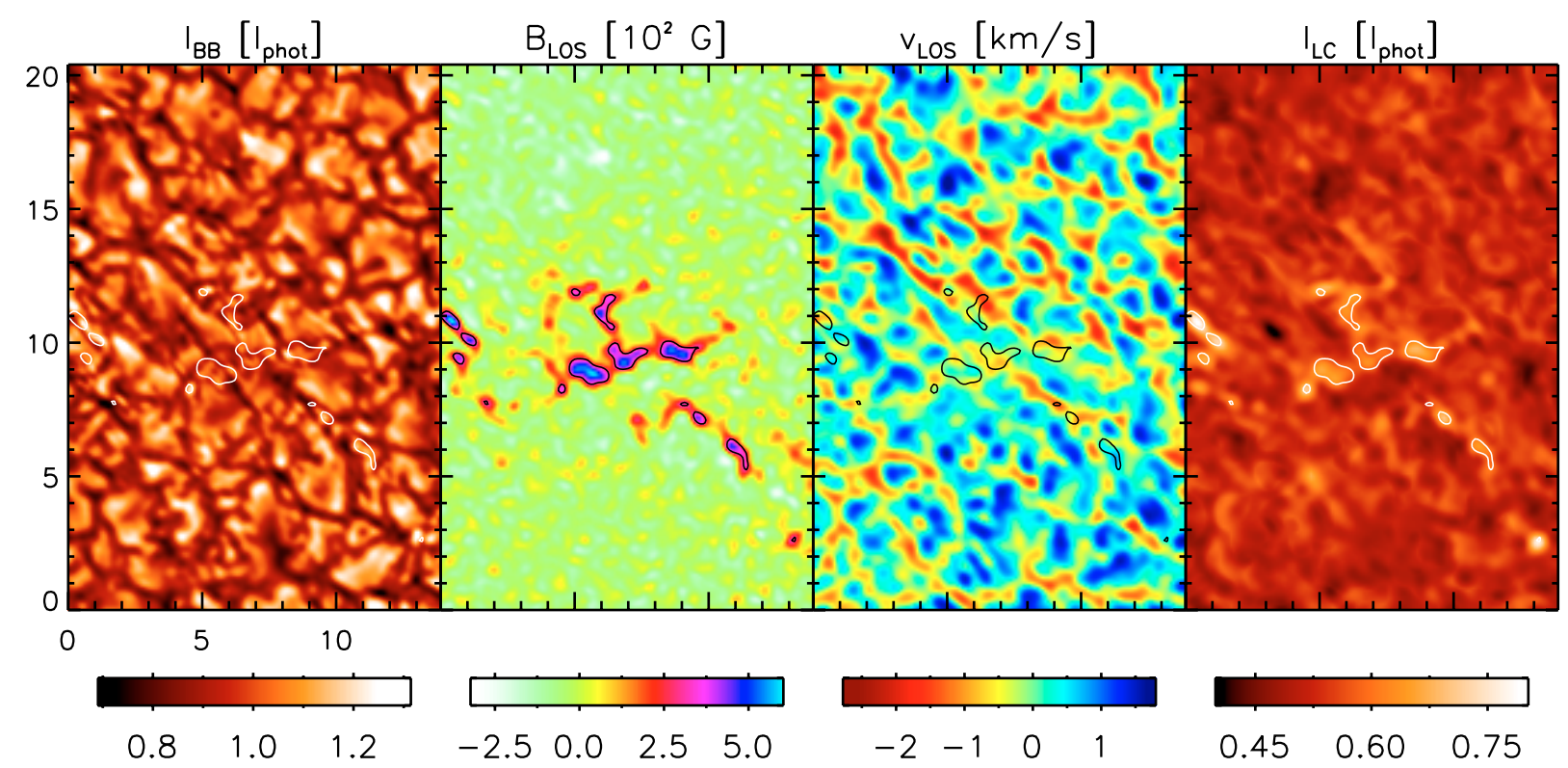

Fig. 3. Region 2: The order of the images is the same as in Fig. 2; in addition the spatial dimensions are similar to those of Region 1. This FOV contains no apparent dark structures, but some network structures are visible in the line core image. The contours show an iso- $B_{\text {LOS }}$ value of 250 G. As in Fig. 2, the tickmarks indicate $1^{\prime \prime}$ and show the measurements performed for the Fe $\mathrm{I} \lambda 6301.5 \AA$ line.

\section{Data processing}

\subsection{Reconstruction of the data}

The broadband reconstruction is based on a program code (de Boer 1996) using speckle interferometric techniques described in Weigelt (1977), Weigelt \& Wirnitzer (1983), and von der Lühe (1984). The resulting broadband image serves as input for the ensuing narrowband reconstruction.

The reconstruction of the narrowband data is done by a "quasi" speckle restoration (Krieg et al. 1999) and the applied code is described in Janßen (2003). However, the determination of the COG (see below) and the computation of the physical quantities $v_{\mathrm{LOS}}$ and $B_{\mathrm{LOS}}$ was enhanced so that the calculation took less time.

\subsection{Determination of maps}

We use the COG method (see Semel 1967; Rees \& Semel 1979) to determine the LOS components of the Doppler velocity and the magnetic field strength. Values obtained by this method are affected by errors of about $10 \%$ to $12 \%$ (cf. Rees \& Semel 1979; Cauzzi et al. 1993), however, the COG method is 
appropriate for computing these quantities as long as the line formation height is kept in mind and therefore, it is a rather reliable method (Uitenbroek 2003).

The narrowband images are smoothed over a $3 \times 3$ pixel boxcar in the spatial domain. The reason is that we do not expect the resolution to be considerably better than 0.3 . Afterwards, the Stokes $I$ and $V$ maps are computed by summation and subtraction of the Stokes $I \pm V$ line profiles, respectively. Furthermore, a second order flatfield was applied to the Stokes $V$ maps as introduced by Sánchez Almeida \& Martínez Pillet (1994), though we use flatfield images instead of data images. Then the positions of the COG of the two Zeeman split line components $I+V$ and $I-V$ were computed for each pixel in the FOV. From these the magnetic field value and the Doppler velocity were computed. The zero velocity was arbitrarily set by subtraction of the mean velocity. As for Region 1, we did not exclude the pore from the averaging process since the velocities occurring within the pore are expected to be close to zero and the ratio of the pore area to the total area is quite small $(<4 \%)$. Hereafter, we always refer to magnetic field strength as the vertical component of the magnetic field.

Moreover, line core intensities, $I_{\mathrm{LC}}$, were determined by computing the minima of the observed Stokes $I$ line profiles without interpolating the line core. This was done since the interpolation is affected by numerical errors and therefore does not return the intrinsic shape of the line core. Thus, we derived the COGs of the temperature response functions (cf. Fig. 1) which provide an indication of the distance to the COG of the continuum formation heights.

The respective noise levels of the broadband images, the LOS magnetic field maps, and the Doppler velocities were determined by means of power spectra, which provide the following error estimates: the broadband intensities are accurate within $\pm 0.02 I_{\text {phot }}$, the Doppler velocities within $\pm 0.08 \mathrm{~km} \mathrm{~s}^{-1}$, and the magnetic field noise level is $\pm 55 \mathrm{G}$.

The two Regions are displayed in Figs. 2 and 3; from left to right we present a broadband image $I_{\mathrm{BB}}$ normalized to the mean photospheric intensity $\left(I_{\text {phot }}\right)$, the associated LOS magnetic field map, $B_{\mathrm{LOS}}$, in units of $10^{2} \mathrm{G}$, the Doppler velocity map $\left(v_{\mathrm{LOS}}\right.$, in $\left.\mathrm{km} \mathrm{s}^{-1}\right)$, and the respective line core map, $I_{\mathrm{LC}}$ (in units of $I_{\text {phot }}$ ). The ticks are at distances of $1^{\prime \prime}$ and the colorbar values are given in the respective physical units displayed above each image. The contours denote an isophote of $0.77 I_{\text {phot }}$ for Region 1 and a constant magnetic field strength of $250 \mathrm{G}$ for Region 2.

The direction of downflows is represented by values below zero, while upflows have positive signs. All coordinates given as $(x, y)$ are in units of arcseconds and correspond to Figs. 2 and 3 , respectively.

\section{Results}

\subsection{Line core vs. velocity scatter plots}

Figure 4 presents 9 scatter plots in which the line core intensity - normalized to the continuum intensity - is plotted as a function of the Doppler velocity of the plasma. Each plot is scaled according to a constant range of $B_{\mathrm{LOS}}(\approx 204 \mathrm{G}$ wide), starting in the upper left corner with the most negative $B_{\mathrm{LOS}}$ values which increase to the right and towards the bottom. We selected the seven best scans with $r_{0}>10 \mathrm{~cm}$ of the first time series containing Region 1, since the scatter spreads for smaller values of the Fried parameter, i.e. poorer seeing conditions.

Figure 4a: the site of the strongest magnetic field is not necessarily located in the darkest regions of the pore but rather at its edges. Depending on which of the best images one chooses, the strongest $B_{\mathrm{LOS}}$ appears in the pore (preferentially at its visual boundary; at position $(7,10)$ in Fig. 2), in a single small micropore (visual diameter of $0 .{ }^{\prime} 8$, at a distance of $1^{\prime \prime}$ from the pore at $\left.(4,8.5)\right)$, in the middle of the protopore at $(4,5)$, and in the center of another magnetic structure (visual diameter 0.'5) next to the protopore at $(5,6)$. Moreover, there are points referring to the very center of the pore exhibiting $0.38 I_{\text {phot }}<I_{\mathrm{LC}}<0.46 I_{\text {phot }}$, and there exist points with $I_{\mathrm{LC}}>0.46 I_{\text {phot }}$ which cover an area outside the visible range of the protopore. The velocity is preferentially downward and does not exceed $-1 \mathrm{~km} \mathrm{~s}^{-1}$, which in the case of the protopore suggests a further growing and in the event of the pore indicates areas of remnant downward flows (Brants 1985). The different line core intensities of the two regimes indicate that the lateral radiative transfer is efficient enough to increase $I_{\mathrm{LC}}$ of the smaller protopore compared to the $I_{\mathrm{LC}}$ of the pore;

Figure $4 \mathrm{~b}$ : these points represent small areas around the previously mentioned regions outlining them in thin stripes. There is a clear separation between pixels belonging to the pore $(7,11)$ and the protopore $(4,5)$ regarding the $I_{\mathrm{LC}}$;

Figures $4 \mathrm{c}$ and $\mathrm{d}$ : this separation vanishes, since the number of points increases and $I_{\mathrm{LC}}$ is shifted, on average, towards slightly higher values. These boundary regions of the pores have $\left|B_{\mathrm{LOS}}\right|<1 \mathrm{kG}$; however, the velocity direction is still mainly downward, thus we detect downflows at the edges of magnetic structures with a maximum of $-1 \mathrm{~km} \mathrm{~s}^{-1}$;

Figure 4e: The shift to higher average $I_{\mathrm{LC}}$ values continues. The shape is rather vertical for points with $0.5 I_{\mathrm{phot}}<I_{\mathrm{LC}}<$ $0.65 I_{\text {phot }}$, however, points below this $I_{\mathrm{LC}}$ follow a somewhat linear relation, although an anticorrelation between $I_{\mathrm{LC}}$ and $v_{\mathrm{LOS}}$ exists;

Figure 4f: a change in the distribution of the points from vertical to more circular is evident as the velocity now ranges from $-1.9 \mathrm{~km} \mathrm{~s}^{-1}$ to about $0.75 \mathrm{~km} \mathrm{~s}^{-1}$. It is mainly the intergranular lanes and the associated downflows that are detected here. However, this plot marks primarily the magnetic boundary of the pore which is located outside the edge of visible magnetic structures (cf. Keppens \& Martínez Pillet 1996);

Figure 4g: this scatter plot marks the boundary between the granular regions and the dark magnetic structures in the broadband image with the exception of the filamentary magnetic channels (e.g. the structure at $(9,18)$ in the magnetic map of Fig. 2). Additionally, there are points in the line core image which appear bright though they cannot be identified as bright areas in the broadband image. Therefore, this property makes them indistinguishable from the granular pattern surrounding these structures. Mainly, 

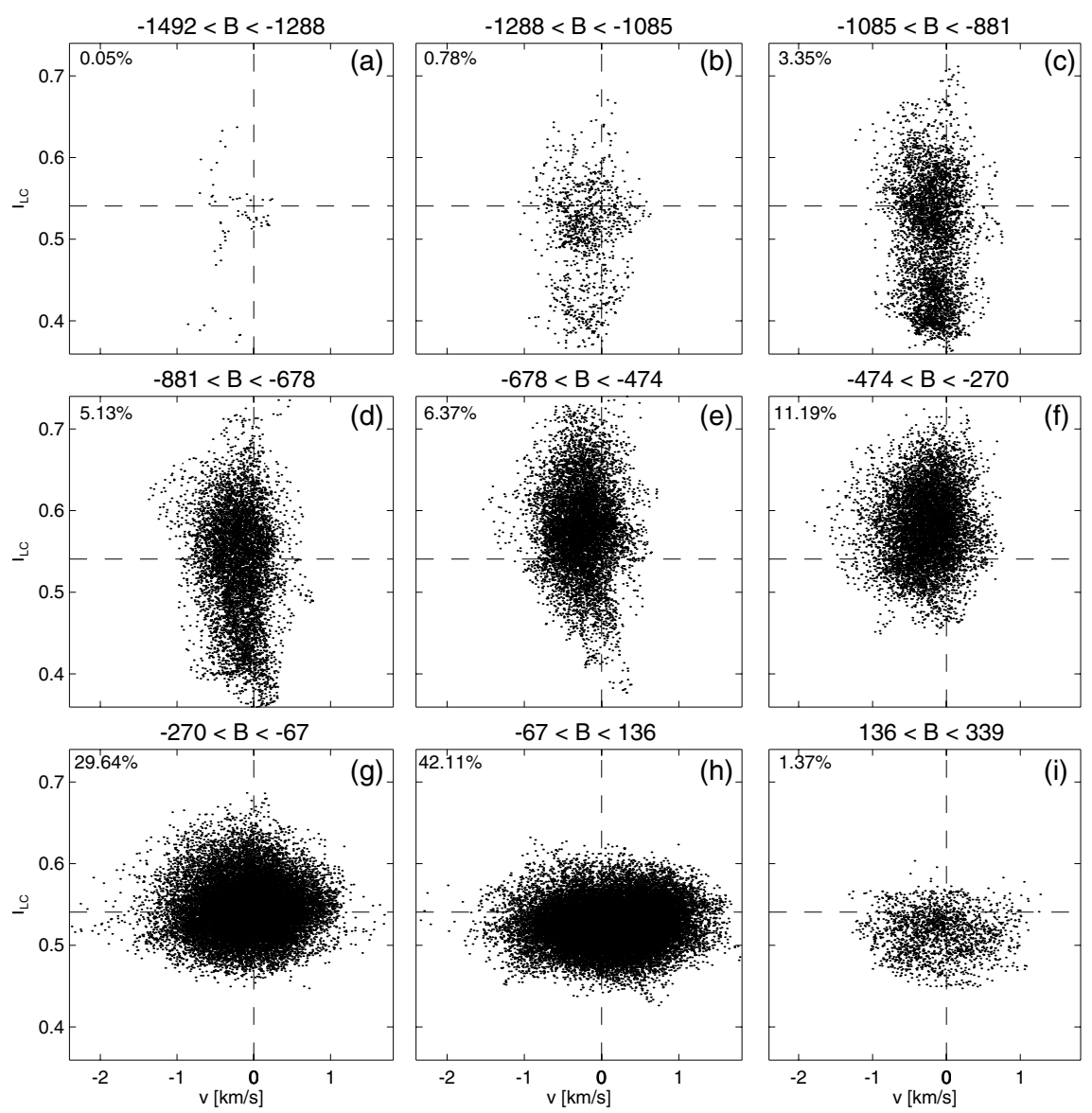

Fig. 4. Scatter plots of the Doppler velocity as a function of the line core intensity for Region 1 . The plots are divided into 9 field strength bins $\left(\approx 204 \mathrm{G}\right.$ wide) for the best images of the time series. The dashed vertical line denotes $v_{\mathrm{LOS}}=0 \mathrm{~km} \mathrm{~s}^{-1}$, i.e. the average $v_{\mathrm{LOS}}$ of the FOV, while the mean of $I_{\mathrm{LC}}$ is symbolized by the horizontal dashed line at $I_{\mathrm{LC}}=0.53 I_{\mathrm{phot}}$. In the upper left corner of each plot the fraction of points in percent are given. Details are presented in the text.

the granular motion starts to dominate the shape of this scatter plot where the distribution of points is now rather circular and is dispersed more uniformly around the zero velocity and the average mean $I_{\mathrm{LC}}\left(0.53 I_{\text {phot }}\right)$;

Figure 4h: the scatter now appears as an elliptical distribution representing the granulation pattern, which is thus dominated by the up- and downward directed motions. The field strengths are $\left|B_{\mathrm{LOS}}\right| \lesssim 135 \mathrm{G}$, i.e. the plasma is quasi fieldfree (since the noise level is at $\left|B_{\mathrm{LOS}}\right| \lesssim 55 \mathrm{G}$ ) and $I_{\mathrm{LC}}$ is distributed slightly below the mean of $0.53 I_{\text {phot }}$. The sign of the magnetic field changes from negative to positive;

Figure 4i: although only a small number of points has positive field strength in Region 1 they can be identified in the magnetic field map (cf. Fig. 2), though not in the line core image, since their $I_{\mathrm{LC}}$ is similar to the mean.

\subsection{Line core vs. field strength scatter plots}

Similar to Fig. 4, we present 9 scatter plots in Fig. 5 where we display the magnetic field strength as a function of $I_{\mathrm{LC}}$ scaled into equidistant velocity ranges of approximately $0.47 \mathrm{~km} \mathrm{~s}^{-1}$ width. Again we selected the seven best images with $r_{0}>$ $10 \mathrm{~cm}$ of Region 1.
Figures 5a-c: the scatter plots comprise small sets of points which belong to strong downflow regions with intermediate field strengths between $-550 \mathrm{G}<B_{\mathrm{LOS}}<150 \mathrm{G}$. A rather linear relation is established, i.e. the brightness in the line core is well correlated with the strength of the magnetic field. One part of these points occurs in intergranular lanes (points with $B_{\mathrm{LOS}} \approx|150| \mathrm{G}$ and $I_{\mathrm{LC}} \approx 0.5 I_{\text {phot }}$ ), whereas the other fraction is located adjacent to the edge of the magnetic boundary of the protopore (e.g. $(3,4),(5.5$, 5.5)). More points are added to the previously established linear states, expanding $I_{\mathrm{LC}}$ to higher and lower values;

Figure 5d: the panel shows that this linear dependence of the magnetic field is disturbed. Abruptly, there appear points with field strengths around $-1000 \mathrm{G}$ and very low $I_{\mathrm{LC}}$. These are not located within the pore as one would suspect but in-between the pore and the protopore in the area of abnormal granulation $(3,9)$. Here we find points which have $B_{\mathrm{LOS}}$ values similar to the ones measured in the pore; however, they are connected with downflows of several hundreds of meters per second. These downflow channels should appear in the vicinity of the pore and protopore as predicted by theoretical models (e.g. Deinzer et al. 1984; Knölker \& Schüssler 1988; Stein et al. 2002) and measured by Keil et al. (1999) and Hirzberger (2003). However, 

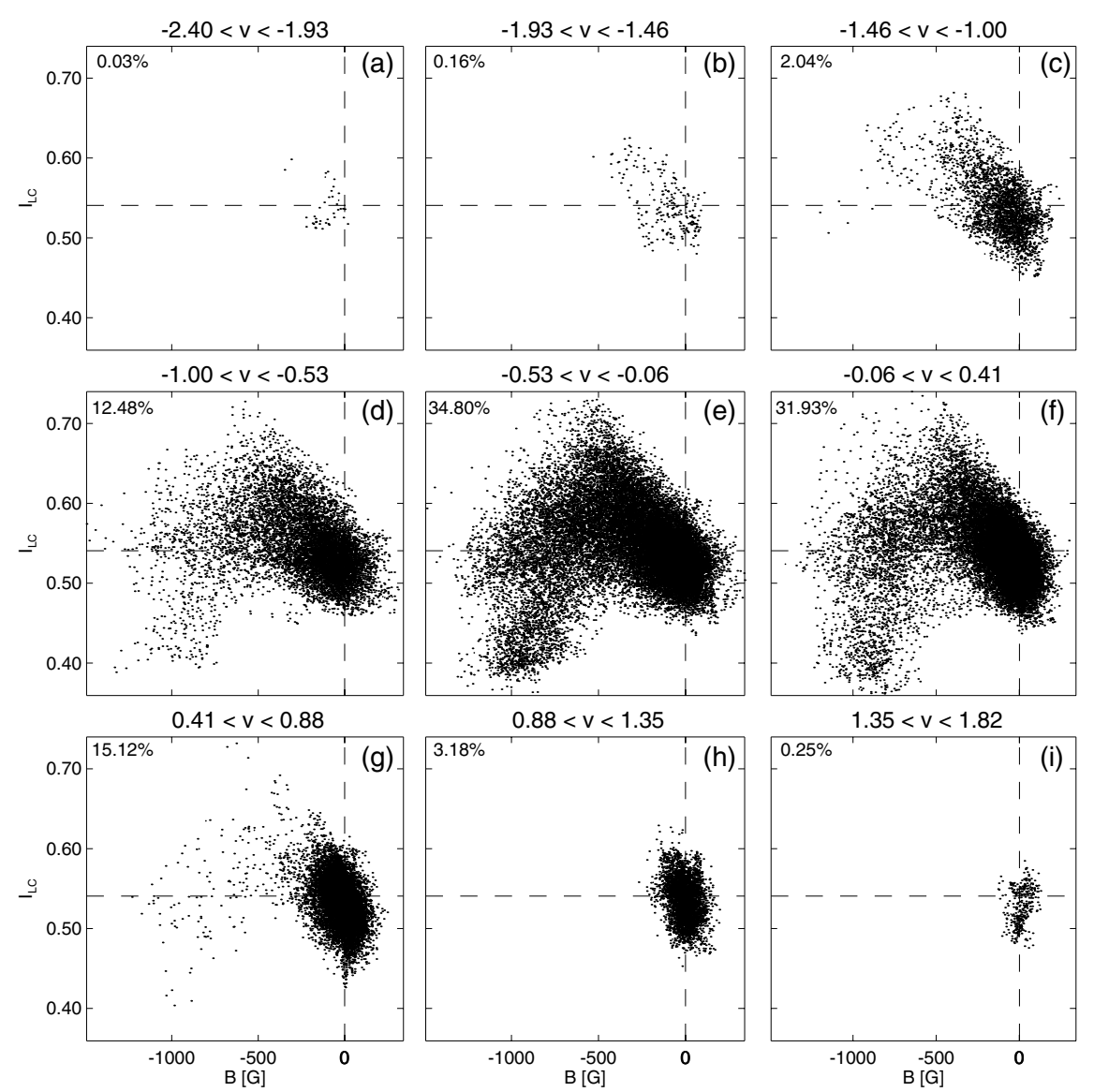

Fig. 5. The line core intensity plotted vs. magnetic field strength for Region 1 and cut into 9 equidistant velocity ranges $\left(0.47 \mathrm{~km} \mathrm{~s} \mathrm{~s}^{-1}\right.$ width). Again, the horizontal line displays the mean $I_{\mathrm{LC}}$, whereas the vertical line exhibits $B_{\mathrm{LOS}}=0 \mathrm{G}$. The fraction of points is printed in the upper left corner of each plot. Refer to the text for details.

they occur mostly approximately 1 " away from the visible boundaries of these magnetic structures. These downflow channels do not completely surround the dark structures, though;

Figure 5e: these points belong to the following areas in Region 1: magnetic edge of the pore (several $10^{2} \mathrm{G}$, mean $I_{\mathrm{LC}} ;(3,11)$ ), the abnormal granular region (up to $1 \mathrm{kG}$, preferentially above the mean $\left.I_{\mathrm{LC}} ;(4,7)\right)$, the magnetic boundary of the protopore (several $10^{2} \mathrm{G}$, above the mean $I_{\mathrm{LC}} ;(4,4)$ ), to the very edges of the granules (some $10^{2} \mathrm{G}$, low $\left.I_{\mathrm{LC}} ;(12,8)\right)$, and the pore $\left(B_{\mathrm{LOS}}>1 \mathrm{kG}, I_{\mathrm{LC}} \simeq\right.$ $\left.0.4 I_{\text {phot }} ;(7,11)\right)$;

Figure 5f: the previous described situation continues. Moreover, points within the pore are added to the scatter with very low $I_{\mathrm{LC}}$ and high $\left|B_{\mathrm{LOS}}\right|$. There are additional contributions to the scatter from points lying in intergranular lanes as well as on granules (bulk of points with $\left.\left|B_{\mathrm{LOS}}\right| \lesssim 200 \mathrm{G}\right)$;

Figure $5 \mathrm{~g}$ : here the convective motions of the gas dominate the scatter, therefore the slope of the bulk of points $(-250 \mathrm{G} \lesssim$ $B_{\mathrm{LOS}} \lesssim 250 \mathrm{G}$ ) becomes steeper as strong magnetic points become less numerous. Some of these are located partly at the edge of the pore, others are found in the pore, indicating that the convective motion of the plasma in the pore is not yet completely inhibited by the magnetic field;
Figures 5h and i: the last two plots display the upward convective motion of the plasma within the granules. The field strength distribution becomes narrower and the scatter is oriented nearly vertically. In addition, $I_{\mathrm{LC}}$ is spread around the mean value. The field strength does not exceed $|100| \mathrm{G}$.

\subsection{How results depend on the observed target}

In Fig. 6 we display plots of the LOS field strength vs. the line core intensity for both regions. The brightest points were selected applying again $I_{\text {bright }}>\bar{I}_{\mathrm{LC}}+2.75 \cdot \sigma_{\mathrm{I}}$ (measured in $I_{\text {phot }}$ ) to the best images of each region. We performed linear fits (solid lines) to the cloud of points in order to measure the trend of $\mathrm{d} B / \mathrm{d} I_{\mathrm{LC}}$. The top axes show the line core brightness excess $\delta I_{\mathrm{LC}}=I_{\mathrm{LC}} / \bar{I}_{\mathrm{LC}}$, where $\bar{I}_{\mathrm{LC}}$ is the mean line core intensity of the respective Region. Moreover, the sign of the magnetic field of Region 2 was reversed for display purposes. In the left panel (Region 1) the spreading of the points is larger than for Region 2 (right panel). Mainly, the dispersion in the left scatter plot is caused by points with $I_{\mathrm{BB}} \approx 1.15 I_{\text {phot }}$, which are located about 2..5 away from the visual boundary of the pore. Thus, for the network Region 2 the correlation of the $I_{\mathrm{LC}}$ brightness and the strength of the LOS magnetic field is better than in Region 1 . The spreading of the points in the latter area is caused by highmagnetic points $\left(B_{\mathrm{LOS}}<-800 \mathrm{G}\right)$. On 

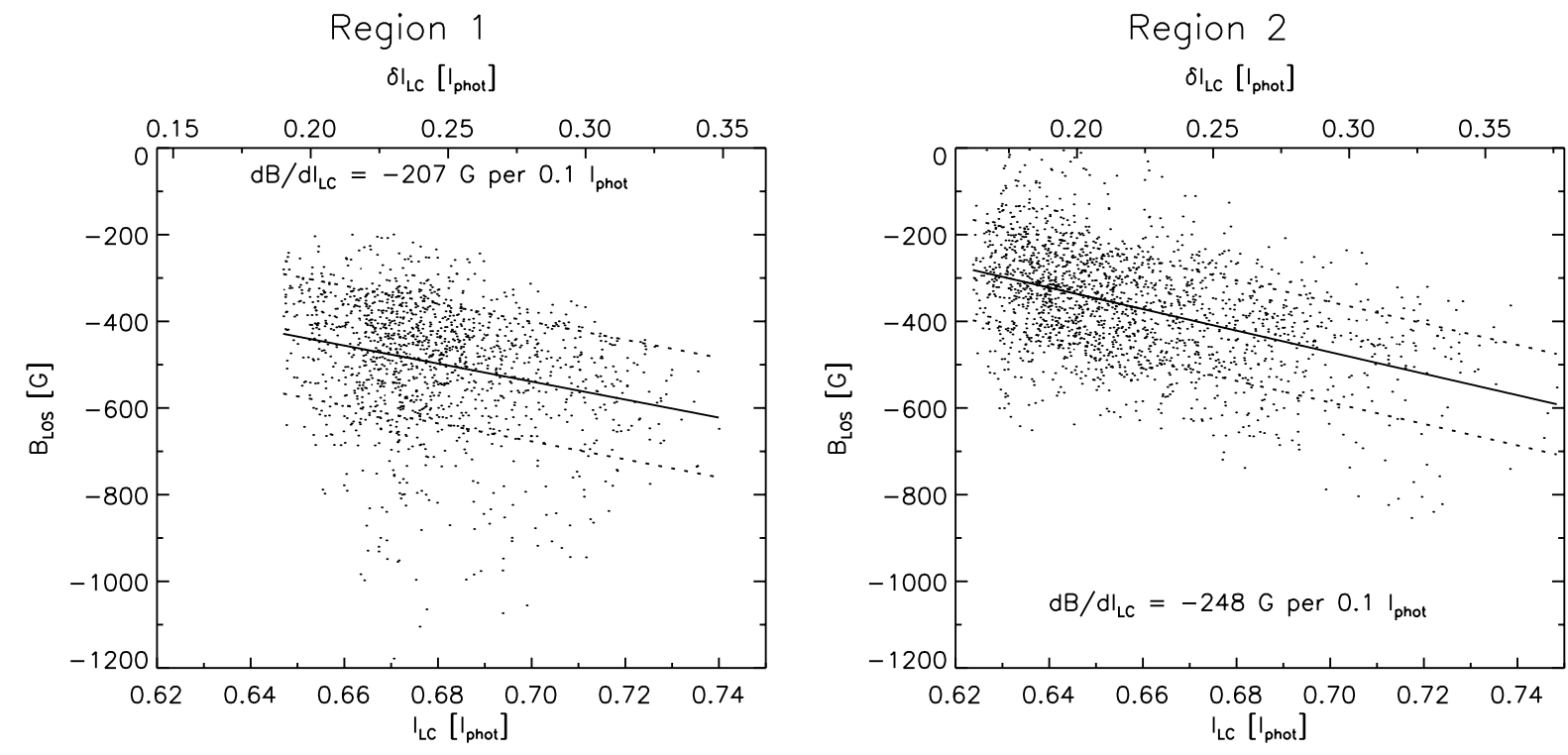

Fig. 6. The brightest line core points vs. the LOS magnetic field component. The left panel shows the distribution computed for Region 1 , the right panel displays the dispersion for Region 2 (the sign of $B_{\mathrm{LOS}}$ is reversed for clarity). The top axes show the line core brightness excess. Linear least squares fits to the scatter are drawn as solid lines, their $1 \sigma$ range is displayed as dashed lines.

the one hand, there exist points $\left(I_{\mathrm{LC}} \approx 0.68 I_{\mathrm{phot}}\right)$ with $B_{\mathrm{LOS}}$ ranging from $-1100 \mathrm{G}$ to $-250 \mathrm{G}$, on the other hand there are points with $B_{\mathrm{LOS}}=-1000 \mathrm{G}$ with $0.66 I_{\mathrm{phot}} \lesssim I_{\mathrm{BB}} \lesssim 0.71 I_{\mathrm{phot}}$; this is the above mentioned spreading.

The value of the gradient $\mathrm{d} B / \mathrm{d} I_{\mathrm{LC}}$ for the selected points of Region 1 is $(-207 \pm 14) \mathrm{G}$ per $0.1 I_{\text {phot }}$, which is somewhat lower than for those of Region 2 , where $\mathrm{d} B / \mathrm{d} I_{\mathrm{LC}}=$ $(-248 \pm 12) \mathrm{G} / 0.1 I_{\text {phot }}$. However, since both slopes are negative there is an intrinsic trend for the brightest line core points to be magnetically independent of the selected solar region.

Since we want to find out the properties of magnetic features we computed the mean values of $I_{\mathrm{BB}}, I_{\mathrm{LC}}$, and $v_{\mathrm{LOS}}$ for the absolute values of $B_{\mathrm{LOS}}$ in $25 \mathrm{G}$ bins following TTT92. The curves are normalized to the value of the non-magnetic bin (1.0 $I_{\text {phot }}$ for $I_{\mathrm{BB}}$ and $I_{\mathrm{LC}}, 0.0 \mathrm{~km} \mathrm{~s}^{-1}$ for $\left.v_{\mathrm{LOS}}\right)$. Figure 7 displays the curves for Region 1 (plus signs) and Region 2 (squares). The upper left panel exhibits the mean broadband intensity, the mean line core intensity is shown in the upper right panel, and the respective curves for the Doppler velocity are displayed in the lower left panel. Error bars - corresponding to the $1 \sigma$ standard deviation of the data - are indicated for the respective quantities. For clarity reasons we give only every fifth and fourth error bar for the data points of Region 1 and Region 2, respectively, since the standard deviations are similar for nearly all points. Furthermore, for the sake of completeness we present in the lower right panel the histograms of the number of points for each bin.

First of all, we specify the results displayed in Fig. 7 for Region 1 and 2 and then we point out the differences to the works of TTT92 and MMV96. For Region 1 we find that

1. the mean values diminish until they reach a local minimum at approximately $200 \mathrm{G}$, increase again to a value right below $1.0 I_{\text {phot }}(400 \mathrm{G}$ to $500 \mathrm{G})$, and decrease nearly linearly to $0.73 I_{\text {phot }}$ at $1 \mathrm{kG}$. For $\left|B_{\mathrm{LOS}}\right|>1 \mathrm{kG}$ the slope of the curve is slightly decreasing. However, this effect might be caused by the smaller number of high-magnetic points available for the statistical averaging process;

2. up to $\left|B_{\mathrm{LOS}}\right|=400 \mathrm{G}$ there is an increase in the mean line core brightness (10\%) which is only slightly decreasing to about $600 \mathrm{G}$ and diminishes linearly from this value to cross the average $I_{\mathrm{LC}}$ at $800 \mathrm{G}$ down to a minimum at $1000 \mathrm{G}$. For values beyond, due to the small number of points it is not possible to decide if the curves approach an expected constant value of approximately $0.95 I_{\text {phot }}$ or slightly rise again;

3. a conspicuous decrease of $v_{\text {LOS }}$ with field strength of less than $400 \mathrm{G}$ is apparent, then the mean Doppler velocity rises again, however, at a smaller rate. Thereafter, the previously mentioned scatter is again obvious, though there is a slight decrease of the mean Doppler velocity at higher $B_{\text {LOS. }}$

As to the mean curves of Region 2 we recognize that

1. the shape of the mean broadband intensity curve is similar to that of Region 1 up to $\left|B_{\mathrm{LOS}}\right|=300 \mathrm{G}$, however, thereafter $\bar{I}_{\mathrm{BB}}>1.0 I_{\mathrm{phot}}$ for the remaining bins. Because of the nearly identical run of the two curves for $\left|B_{\mathrm{LOS}}\right|<300 \mathrm{G}$, and as the minimum of $\bar{I}_{\mathrm{BB}}$ is around $150 \lesssim\left|B_{\mathrm{LOS}}\right| \lesssim 200 \mathrm{G}$, we identify these as internetwork fields predominantly located in the intergranulum (e.g. Livingston \& Harvey 1975; Keller et al. 1994; Stenflo et al. 1998; Lin \& Rimmele 1999; Domínguez Cerdeña et al. 2003);

2. there is an excess of mean line core brightness of nearly $30 \%$ for the last bins. Thus, we do not determine a decrease of the mean $I_{\mathrm{LC}}$ as observed for Region 1. Hence, the brightest line core points have the highest LOS magnetic field strengths. This is consistent with the interpretation of the right plot in Fig. 6;

3 . the decrease of the mean $v_{\mathrm{LOS}}$ bins is steeper and the minimum is located at lower magnetic field strength than in 

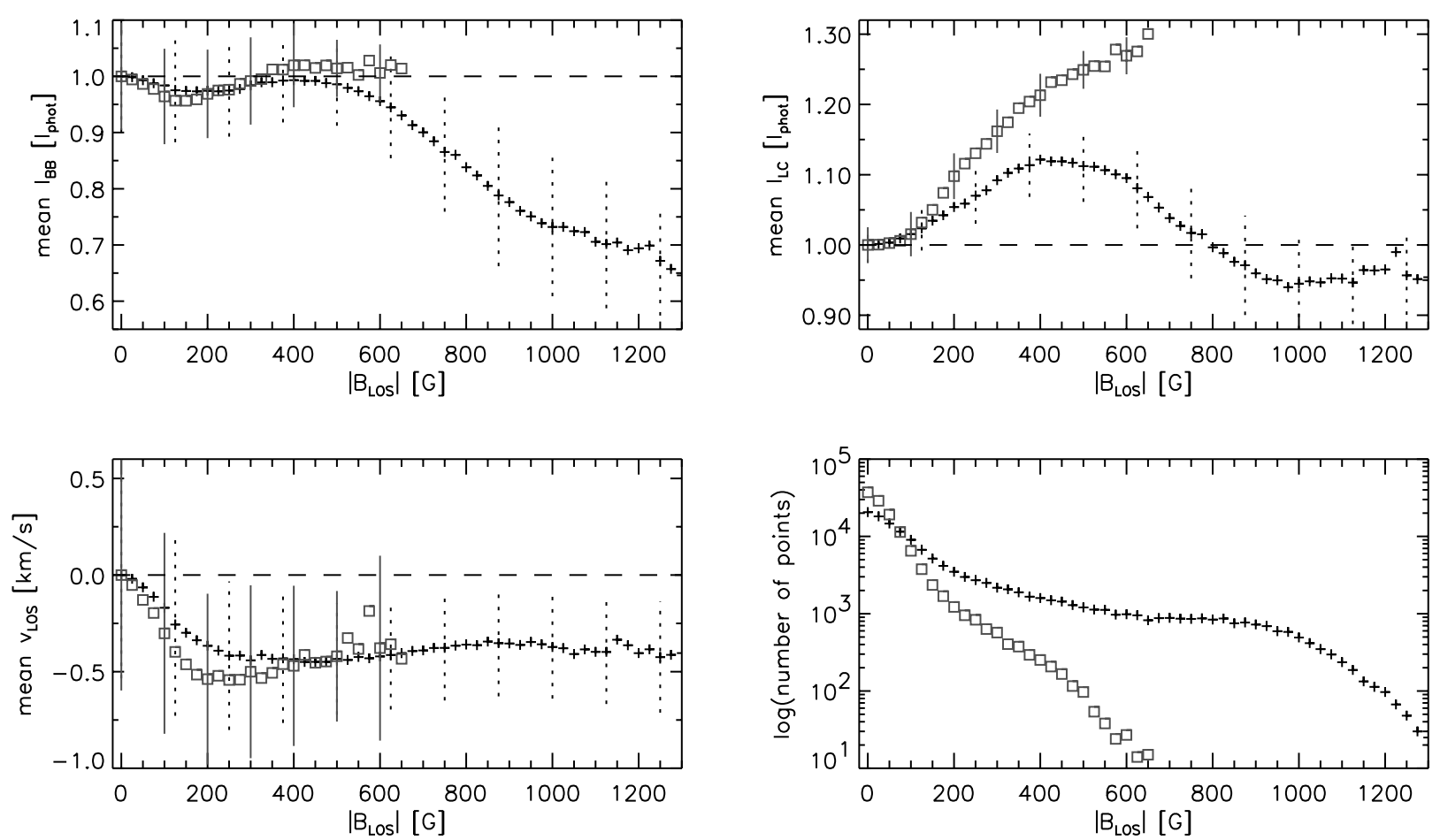

Fig. 7. The mean values of the broadband intensity (top left), the line core intensity (top right) and the Doppler velocity (bottom left) are plotted vs. the absolute value of the LOS magnetic field in bins of $\Delta B=25 \mathrm{G}$. The bottom right plot shows the number of points over which we averaged the respective quantity. Plus signs denote values computed for Region 1 ( $1 \sigma$ errors are indicated by dotted lines) and squares show the results for Region 2 (respective error bars are presented as solid lines). Dashed lines display the normalized mean value of the respective quantity.

Region 1, which is another indication for internetwork magnetic fields in this FOV, since they occur in the intergranular lanes and are thus associated with downflows. Moreover, the Doppler velocities rise more strongly, but are significantly scattered.

The histograms (lower right plot in Fig. 7) give an impression of how many points contribute to each $25 \mathrm{G}$ bin. The number of points in the Region 1 histogram reaches a plateau for $400 \mathrm{G} \lesssim$ $\left|B_{\mathrm{LOS}}\right| \lesssim 1000 \mathrm{G}$. Beyond this the number decreases rapidly, due to the fact that the pore area is small compared to the total area of the FOV. Since only granular structures are visible in Region 2 there is a steeper decrease in number than in Region 1.

As for Region 1, the shapes of the calculated mean curves approximately resemble Fig. 16 to 18 of TTT92 and Fig. 6 of MMV96. Since the heliocentric angle $\mu$ is 0.98 for Region 1 and nearly 1.00 for Region 2, the shape of the curves displayed in Fig. 7 resembles more closely those of TTT92 because their FOV is located at a similar $\mu$. As opposed to these results, MMV96 have observed at a heliocentric angle of 0.88 and they explain the discrepancy with the work of TTT92 by the different $\mu$ of the observations and discriminative calibration of the magnetic field.

Since we analyze two independent sets of data of the solar photosphere, and since the shapes of the averaged curves of Fig. 7 are conspicuously varying, we claim that the shape of the mean curves strongly depends on the selected solar region. This can be verified by a comparison to the distribution of Region 2, which is a purely granular region far away from the (magnetic) influence of an active region and without any obvious dark structure visible in the broadband continuum. Because different solar regions may contain different types of magnetic features, it is dangerous to infer general correlations between magnetic field, velocity, and broadband intensity from the examination of a single solar area.

\subsection{Estimation of flux tube bending}

In order to gather information about the vertical geometrical configuration of flux tubes (apparent as bright points in line core images) we computed the position of the maximum intensity in the line core map and the position of the closest maximum in the magnetic field map. To this end we determined the radial distance $r$ (in arcseconds) of these two values using $r=+\left(\left(x_{\mathrm{LC}}-x_{\mathrm{MAG}}\right)^{2}+\left(y_{\mathrm{LC}}-y_{\mathrm{MAG}}\right)^{2}\right)^{1 / 2}$, with $x$ and $y$ as the position of the maxima in the line core (index LC) and magnetic field strength (index MAG) images, respectively. We computed the radial distances only for bright points fulfilling the following line core intensity criterium: $I_{\text {bright }}>\bar{I}+2.75 \cdot \sigma_{\mathrm{I}}$, where $I_{\text {bright }}$ is the intensity of the bright point, $\bar{I}$ is the mean value of the line core image, and $\sigma_{\mathrm{I}}$ its standard deviation. The factor 2.75 was chosen such that the thus selected bright points are intrinsically bright in the line core images compared to the mean; in addition it ensures that there is a sufficiently large number of points for the desired statistics. This criterium ensures a more seeing-independent selection of bright points than a pure visual inspection. The $I_{\mathrm{LC}}$ intensity maximum of each 

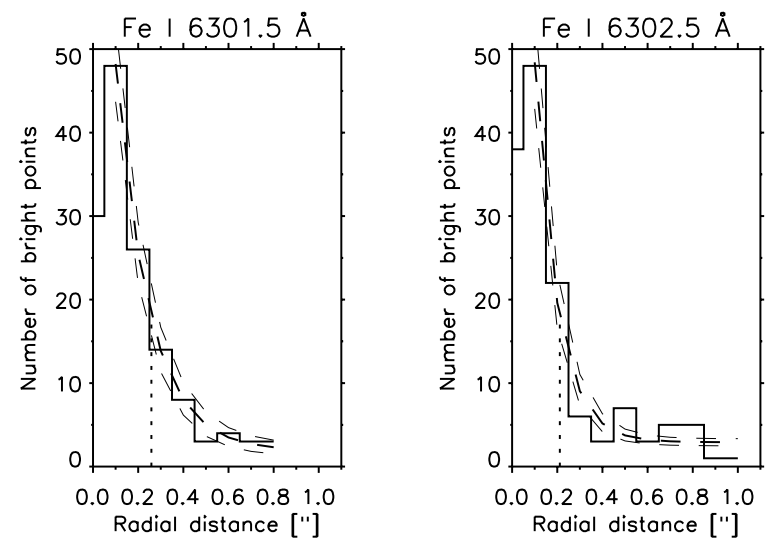

Fig. 8. Histogram of radial distances, in arcseconds, computed from the maximum line core intensity to the maximum of the appropriate local maximum field strength. The exponential fit is indicated as a dashed line (with the $1 \sigma$ errors indicated as small dashed lines) while the $1 / e$-distance is represented as a dotted line.

bright point was taken as the center of a square box of 2 '. 1 width and within this box the location of the $B_{\mathrm{LOS}}$ maximum was computed.

In Fig. 8 we present histograms of this radial displacement (solid lines), an exponential fit (dashed curves) and the $1 / e$ distance (vertical dotted lines) for the Fe I $26301.5 \AA$ (left) and the Fe I $\lambda 6302.5 \AA$ line (right). The number of bright points decreases exponentially in both cases, however, the curve of the Fe I $\lambda 6302.5 \AA$ line drops more steeply and continues flatter towards stronger bending than in the Fe I $\lambda 6301.5 \AA$ case. This can be explained by the different formation heights of the two line cores $\left(\Delta z_{\mathrm{LC}}=40 \mathrm{~km}\right.$, cf. Table 1$)$ which means - according to a flux tube model - that the diameter of the tube increases with height in order to maintain horizontal pressure equilibrium. The $1 / e$-distance for the Fe I $\lambda 6301.5 \AA 0.26 \pm 0 . ' 007$ is and $0^{\prime} .21 \pm 0 . \prime 013$ for the $\mathrm{Fe} \mathrm{I} \lambda 6302.5 \AA$ line, i.e. there is a significant difference between these two values. This might be attributed to the different line core formation heights of the two lines. The $1 / e$ values are close below our resolution limit. For both wavelengths only a small number of tubes bends more than 0.6 and just 21.6\% (Fe I $\lambda 6301.5 \AA$ ) and $27.3 \%$ (Fe I $\lambda 6302.5 \AA$ ) of the respective line core and magnetic field maxima coincide, i.e. they are vertically orientated.

\section{Conclusions}

We examined two photospheric regions near the disk center, one containing a small pore and a protopore embedded in abnormal granulation, the other is part of the network with no suspicious dark features. By means of the reconstructed Stokes $I \pm V$ line profiles, we applied the COG method to derive the components of the Doppler velocity and the LOS magnetic field strength.

In various recent papers (Keil et al. 1999; Hirzberger 2003; Sankarasubramanian \& Rimmele 2003) downflowing channels at the edge of pores with velocities around $-1 \mathrm{~km} \mathrm{~s}^{-1}$ were reported. In agreement with these findings, we find a downflow of plasma at the edges of the pore nearly around the whole structure (cf. Doppler map in Fig. 2). Within the pore there are mixed $v_{\text {LOS }}$ which also have been reported e.g. by Sigwarth et al. (1999) and Sankarasubramanian \& Rimmele (2003). However, the latter authors relate the internal velocity structure to the magnetic fill fraction of the relevant pore: in a pore with large fill fraction they discover small upflows, whereas a pore with small fill fraction exhibits large downflows. In contrast, Leka \& Skumanich (1998) measure a velocity dispersion for the darkest pore of $\left|v_{\mathrm{LOS}}\right| \leq 0.25 \mathrm{~km} \mathrm{~s}^{-1}$. Our results are in good agreement with these findings. From the existence of mixed velocities in the pore we interpret this as a dynamic behavior of the magnetic elements, thus following the conclusion of Sigwarth et al. (1999).

No rings of downflows surrounding small magnetic flux concentrations (bright points) are present either in Region 1 or in Region 2. Rather, patches of downflow channels around these magnetic structures are apparent. This finding is consistent with those of Rimmele (2004), but does not completely resemble the simulations of e.g. Knölker \& Schüssler (1988), Steiner et al. (1998) and Stein et al. (2002).

Considering the protopore of Region 1, we find that the location of the $B_{\mathrm{LOS}}$ maximum in the best scans is nearly centered within the protopore. However, according to Rimmele (2004) the maximum of $B_{\mathrm{LOS}}$ is not centered during the early stages of micropore formation.

Proceeding with the discussion of the pore in Region 1, we discover that in two of our best scans the maximum of $B_{\mathrm{LOS}}$ is located at the edge of the pore while in the other scans it is found nearly in the darkest central parts. Since the variations of the maximum $B_{\mathrm{LOS}}$ differ on the order of some hundreds of Gauss from one scan to the next (well above the noise level of $55 \mathrm{G}$ ) we conclude that this change is of solar origin; however, we are aware that the magnitude of $B_{\mathrm{LOS}}$ is possibly influenced by an unknown amount of stray light. To investigate this effect we performed a test in which we applied artificial stray light by convolution of the raw data with a certain point spread function (see Kneer \& Mattig 1968) showing that the results only have smaller values of $B_{\mathrm{LOS}}$ and the other physical quantities. However, the shapes of the curves and scatter displayed in Figs. 4-7 are only marginally influenced by additional stray light so that the interpretation of these plots does not change. Furthermore, the data were not corrected for five-minute oscillations, since the time period of the series are too short to allow a meaningful subsonic filtering of the data. Therefore, we assume some residual influence on the scatter plots by this effect.

Determining the correlations of the line core intensity $I_{\mathrm{LC}}$ with the LOS magnetic field magnitude reveals a trend for the detected bright points in Region 1; yet there exist numerous outliers which we relate to the fact that the influence of the pore's magnetic field is present in its environment. These two quantities are rather well associated for the other Region. Although Leka \& Steiner (2001) state that even small magnetic elements have a magnetic canopy, which is the flaringout of the magnetic field with height and associated downflows around the magnetic elements, we are not able to confirm this result for bright points in our data. The downflow annulus mentioned in Leka \& Steiner (2001) is rather an effect of their lower spatial resolution, since several patches of downflow channels 
around a flux tube detected at high resolution are smeared to an annulus if observed at modest spatial resolution. A simple visual comparison of the broadband and the line core images of Figs. 2 and 3 only indicates the existence of a magnetic canopy for small magnetic elements (bright points) due to the larger size of these points in the line core images. However, a lower spatial resolution in the $I_{\mathrm{LC}}$ images as compared to the broadband images can contribute to this enlargement.

The values of our rough estimate of flux tube bending in the photosphere $(<0$ '. 3 for nearly two-thirds of analyzed points) confirms the results obtained by numerical simulations (Steiner et al. 1998, detect horizontal displacements up to $0 .{ }^{\prime} 83$ ) if one keeps in mind the small height range covered by our observations. Muller et al. (2000) find that the correspondence of magnetic elements and associated line core bright points does not exceed 0.5 which they also relate to the swaying of flux tubes. Moreover, the spatial dynamics of flux concentrations seems to be closely related to the convective motions of the surrounding granulation (Sigwarth et al. 1999). There is, however, a great discrepancy in the range of continuum brightness $\delta_{\mathrm{c}}=I_{\mathrm{c}}^{\mathrm{m}} / I_{\mathrm{c}}^{\mathrm{qs}}\left(I_{\mathrm{c}}^{\mathrm{m}}\right.$ is the intensity of the magnetic feature, $I_{\mathrm{c}}^{\mathrm{qs}}$ the intensity of the non-magnetic quiet Sun) of small scale magnetic features at disk center: TTT92 propose $\delta_{\mathrm{c}}<1$ for all magnetic features, Solanki \& Brigljevic (1992) give values in the range of 1.1-1.5 for network bright points and $0.7-0.95$ for plages, Muller \& Keil (1983) found values of 1.3-1.5 for facular points, and $\delta_{\mathrm{c}}>1.3$ for continuum bright points co-spatial with magnetic fields were reported by Keller (1992). All these values are based on observations. In their simulations Steiner et al. (1998) found values of 1.1-1.6 with respect to the continuum intensity at $5250 \AA$. They associated the increase of brightness with the hot wall exposed to an observer because of the swaying motion of the flux tube. The continuum intensity of small bright magnetic features $\left(\left|B_{\mathrm{LOS}}\right|>200 \mathrm{G}\right)$ in Region 1 varies from 1.07 to 1.30 (mean at 1.11 ). For Region 2 $\left(\left|B_{\mathrm{LOS}}\right|>120 \mathrm{G}\right)$ the respective values range from 1.07 to 1.32 with a mean brightness of 1.13 .

All data points with $B_{\mathrm{LOS}} \lesssim-680 \mathrm{G}$ are located within a Doppler velocity range of $-1 \mathrm{~km} \mathrm{~s}^{-1}<v_{\mathrm{LOS}}<0.5 \mathrm{~km} \mathrm{~s}^{-1}$, independent of the line core brightness (Figs. 4a-d). This finding is similar to the results of Leka \& Skumanich (1998) who examined the dependence of the normalized continuum intensity and the Doppler velocity.

When we compare the two observed Regions we find clear differences for the examined properties (cf. Figs. 6 and 7). Common to both Regions is the tendency of line core bright points to be more strongly magnetic for brighter $I_{\mathrm{LC}}$ although the scatter is large. This means that not every bright point with high $I_{\mathrm{LC}}$ is associated with a magnetic element. This does not confirm the results of Muller et al. (2000) who assign to every bright point a magnetic element, and vice versa. This discrepancy we explain by the higher spatial resolution of our data. Several other authors (Keller 1992; Title et al. 1992; Berger $\&$ Title 1996) find a correlation of the magnetic field with the brightness of points. However, as pointed out above there exists only a trend and not an intrinsic property. The differences displayed in Fig. 7 for the two Regions indicate that there is a strong dependence on the chosen solar region and the heliocentric angle when one examines small scale magnetic features.

Langhans et al. (2002) analyzed $G$-band bright structures in the solar photosphere and found that there are two different classes of bright $G$-band elements: first, the brightness of $G$-band structures with an enhanced contrast - showing downflows in the intergranulum - are linked to a significant weakening of the $\mathrm{CH}$-absorption line. Second, all other bright structures are related to the granulation and their brightness originates from an enhanced continuum intensity. We can confirm these suggestions qualitatively for the examined line core bright points, although we did not analyze $G$-band lines.

However, for more detailed studies (especially by means of high resolution two dimensional spectroscopy) we are in need of larger solar telescopes that are able to work at these small scales and are equipped with the newest technologies.

Acknowledgements. The authors wish to acknowledge financial support by the Austrian Fonds zur Förderung der wissenschaftlichen Forschung (Project No. R11) and are grateful to an anonymous referee for valuable comments. Furthermore, the authors thank M. Sobotka for useful comments on the manuscript. The Vacuum Tower Telescope at the Spanish Observatorio del Teide of the Instituto de Astrofísica de Canarias, Tenerife, is operated by the Kiepenheuer-Institut für Sonnenphysik in Freiburg, Germany.

\section{References}

Bendlin, C., \& Volkmer, R. 1995, A\&AS, 112, 371

Bendlin, C., Volkmer, R., \& Kneer, F. 1992, A\&A, 257, 817

Berger, T. E., \& Title, A. M. 1996, ApJ, 463, 365

Brants, J. J. 1985, Sol. Phys., 95, 15

Cauzzi, G., Smaldone, L. A., Balasubramaniam, K. S., \& Keil, S. L. 1993, Sol. Phys., 146, 207

de Boer, C. R. 1996, A\&AS, 120, 195

Deinzer, W., Hensler, G., Schüssler, M., \& Weisshaar, E. 1984, A\&A, 139,435

Domínguez Cerdeña, I., Sánchez Almeida, J., \& Kneer, F. 2003, A\&A, 407, 741

Dunn, R. B., \& Zirker, J. B. 1973, Sol. Phys., 33, 281

Hirzberger, J. 2003, A\&A, 405, 331

Janßen, K. 2003, Ph.D. Thesis, Göttingen University

Keil, S. L., Balasubramaniam, K. S., Smaldone, L. A., \& Reger, B. 1999, ApJ, 510, 422

Keller, C. U. 1992, Nature, 359, 307

Keller, C. U., Deubner, F.-L., Egger, U., Fleck, B., \& Povel, H. P. 1994, A\&A, 286, 626

Keppens, R., \& Martínez Pillet, V. 1996, A\&A, 316, 229

Kneer, F., \& Mattig, W. 1968, Sol. Phys., 5, 42

Knölker, M., \& Schüssler, M. 1988, A\&A, 202, 275

Koschinsky, M., Kneer, F., \& Hirzberger, J. 2001, A\&A, 365, 588

Krieg, J., Wunnenberg, M., Kneer, F., Koschinsky, M., \& Ritter, C. 1999, A\&A, 343, 983

Langhans, K., Schmidt, W., \& Tritschler, A. 2002, A\&A, 394, 1069

Leka, K. D., \& Skumanich, A. 1998, ApJ, 507, 454

Leka, K. D., \& Steiner, O. 2001, ApJ, 552, 354

Lin, H., \& Rimmele, T. 1999, ApJ, 514, 448

Livingston, W. C., \& Harvey, J. 1975, BAAS, 7, 346

Montagne, M., Muller, R., \& Vigneau, J. 1996, A\&A, 311, 304

Muglach, K., \& Solanki, S. K. 1992, A\&A, 263, 301 
Muller, R., Dollfus, A., Montagne, M., Moity, J., \& Vigneau, J. 2000, A\&A, 359, 373

Muller, R., \& Keil, S. L. 1983, Sol. Phys., 87, 243

Pérez Rodríguez, E., \& Kneer, F. 2002, A\&A, 395, 279

Rees, D. E., \& Semel, M. D. 1979, A\&A, 74, 1

Rimmele, T. R. 2004, ApJ, 604, 906

Sánchez Almeida, J. 1997, ApJ, 491, 993

Sánchez Almeida, J., \& Martínez Pillet, V. 1994, ApJ, 424, 1014

Sankarasubramanian, K., \& Rimmele, T. 2003, ApJ, 598, 689

Schüssler, M., \& Solanki, S. K. 1988, A\&A, 192, 338

Semel, M. 1967, Ann. Astrophys., 30, 513

Sigwarth, M., Balasubramaniam, K. S., Knölker, M., \& Schmidt, W. 1999, A\&A, 349, 941

Solanki, S. K., \& Brigljevic, V. 1992, A\&A, 262, L29

Stein, R. F., Bercik, D., \& Nordlund, Å. 2002, Nuovo Cimento C, 25, 513
Steiner, O. 2000, Sol. Phys., 196, 245

Steiner, O., Grossmann-Doerth, U., Knölker, M., \& Schüssler, M. 1998, ApJ, 495, 468

Stenflo, J. O. 1973, Sol. Phys., 32, 41

Stenflo, J. O., Keller, C. U., \& Gandorfer, A. 1998, A\&A, 329, 319

Stolpe, F., \& Kneer, F. 1997, A\&A, 317, 942

Title, A. M., Topka, K. P., Tarbell, T. D., et al. 1992, ApJ, 393, 782

Topka, K. P., Tarbell, T. D., \& Title, A. M. 1992, ApJ, 396, 351

Uitenbroek, H. 2003, ApJ, 592, 1225

Vernazza, J. E., Avrett, E. H., \& Loeser, R. 1981, ApJS, 45, 635

von der Lühe, O. 1984, Opt. Soc. Am. J., 1, 510

Weigelt, G., \& Wirnitzer, B. 1983, Opt. Lett., 8, 389

Weigelt, G. P. 1977, Opt. Commun., 21, 55

Wiehr, E., Bovelet, B., \& Hirzberger, J. 2004, A\&A, submitted

Zayer, I., Solanki, S. K., \& Stenflo, J. O. 1989, A\&A, 211, 463 